\section{Automedicação em idosos residentes em Campinas, São Paulo, Brasil: prevalência e fatores associados}

\author{
Self-medication in the elderly population \\ of Campinas, São Paulo State, Brazil: \\ prevalence and associated factors
}

${ }^{1}$ Faculdade de Ciências Médicas, Universidade Estadual de Campinas, Campinas, Brasil.

2 Secretaria de Ciência Tecnologia e Insumos Estratégicos, Ministério da Saúde, Brasília, Brasil.

Correspondência P. M. S. B. Francisco Faculdade de Ciências Médicas, Universidade Estadual de Campinas. Rua Tessália Vieira de Camargo 126, Cidade Universitária Zeferino Vaz, Campinas, SP 13083-877, Brasil.

primaria@fcm.unicamp.br

\section{Abstract}

The aim of this study was to evaluate the prevalence and causative factors associated with selfmedication in the elderly and identify the main drugs consumed without prescription. A crosssectional population-based study with stratified clustered two-stage sampling was performed in Campinas, São Paulo, Brazil in 2008 and 2009. Of the 1,515 elderly studied, $80.4 \%$ reported using at least one drug duringthe three days preceding the survey. Of these, $91.1 \%$ reported the use of prescription drugs only and the remainder (8.9\%) reported simultaneous use of prescribed and non prescribed drugs. After adjustment, $a$ negative association between age $\geq 80$ years, hypertension, chronic diseases, use of health services, dental consultations and adherence to a medical plan, and self-medication was found, whereas a positive association was found with per capita income. Dipyrone, acetylsalicylic acid, diclofenac, Ginkgo biloba, paracetamol and homeopathic medicines were among the most used non-prescribed drugs. Pharmaceutical assistance should be provided as a priority tothe elderly, to avoid the misuse of medicines and ensure access to the correct drugs.

Self-medication; Drug Utilization; Pharmacoepidemiology; Helath of the Elderly
Marcelo Antunes de Oliveira 1

Priscila Maria Stolses Bergamo Francisco ${ }^{1}$

Karen Sarmento Costa 1,2

Marilisa Berti de Azevedo Barros 1

\section{Introdução}

O envelhecimento populacional tem gerado novas demandas sociais, econômicas, sanitárias e, diante da importância crescente deste segmento, estudos envolvendo a população idosa constituem um tema emergente nas diversas áreas de conhecimento $1,2,3$.

A morbidade apresentada pela população idosa caracteriza-se pela preponderância de doenças crônicas e múltiplas de longa duração, exigindo acompanhamento, cuidados permanentes e exames periódicos. O idoso utiliza mais serviços de saúde, as internações hospitalares são mais frequentes do que entre adultos e o tempo de ocupação do leito é maior quando comparado a outras faixas etárias 3,4. Ainda, o aumento da prevalência de doenças crônicas com a idade, demanda um maior consumo dos medicamentos, que constituem um dos itens mais importantes da atenção à saúde do idoso 4,5,6,7, e requer, cada vez mais, a racionalidade da terapia medicamentosa $5,8,9$.

São inegáveis os benefícios terapêuticos conseguidos com o uso correto dos medicamentos, no entanto, seu elevado consumo entre os idosos pode acarretar riscos à saúde. Os idosos fazem uso, em média, de dois a cinco medicamentos diariamente 10,11 e são particularmente mais sensíveis aos efeitos adversos, interações medicamentosas e toxicidade $9,12,13$. Na popu- 
lação idosa, estudos apontam a predominância do uso de medicamentos prescritos 5,6,8,9,14,15,16, mas neste segmento etário é comum encontrar prescrições de doses e indicações inadequadas, redundâncias e o uso de medicamentos sem valor terapêutico 8,9,17. Além disso, o consumo de medicamentos sem prescrição de um profissional de saúde habilitado (automedicação) tornase um dos problemas derivados desse uso pela população idosa 15,17.

A automedicação é uma forma de autocuidado à saúde, entendida como a seleção e uso de medicamentos para manutenção da saúde, prevenção de enfermidades, tratamento de doenças ou sintomas percebidos pelas pessoas, sem a prescrição, orientação ou o acompanhamento do médico ou dentista 18 e é uma prática comum na população brasileira 14,19,20,21,22,23. Fatores como a familiaridade com o medicamento, experiências positivas anteriores, a função simbólica que os medicamentos exercem sobre a população, e a dificuldade de acesso aos serviços de saúde, contribuem para a automedicação 14,16,20,24.

A prevalência e os fatores associados à automedicação em idosos vêm sendo investigada por meio de estudos epidemiológicos de base populacional, e os resultados apontam que tal prática varia entre os idosos residentes em diferentes localidades 5,15,19,25,26. Nos Estados Unidos, estudo com amostra representativa da população verificou que $42 \%$ usavam, no mínimo, um medicamento sem receita 26 . No sul da Austrália, Goh et al. 25 encontraram prevalência de $17 \%$ em 2000-2001 e de 35,5\% em 2003-2004. No Brasil, estudo realizado em Bambuí (Minas Gerais) verificou prevalência de $17 \% 15$ e, no Município de Salgueiro (Pernambuco), 60\% dos idosos entrevistados praticavam a automedicação 19 .

No que se refere aos fatores associados à automedicação em idosos, estudos têm apontado maior consumo de medicamentos sem prescrição entre as mulheres 15,19,25,26,27. Em relação à idade, tanto a redução 12,25 quanto o aumento 15 da automedicação entre os mais longevos, foram verificados. Ainda, melhor nível socioeconômico 5,15, filiação a plano de saúde 15,26, maior número de consultas médicas 15 e presença de doenças/condições crônicas 15,19 apresentam associação inversa à automedicação.

Particularmente entre os idosos, as desvantagens da automedicação devem ser consideradas e, entre elas, destacam-se os gastos desnecessários, atraso no diagnóstico e na terapêutica adequados, potenciais riscos de interações com os medicamentos prescritos, resistência bacteriana, reações adversas e intoxicação 19,23,24.

Considerando-se o exposto, o objetivo deste estudo foi estimar a prevalência e fatores associa- dos à automedicação entre idosos residentes no Município de Campinas, bem como identificar os principais fármacos consumidos sem prescrição para essa população.

\section{Métodos}

Estudo transversal de base populacional que incluiu 1.515 idosos (60 anos e mais) não institucionalizados, residentes em área urbana no Município de Campinas no período de 2008 e 2009. Os dados utilizados neste estudo são provenientes do Inquérito de Saúde no Município de Campinas (ISA-Camp), realizado pelo Centro Colaborador em Análise de Situação de Saúde (CCAS) do Departamento de Medicina Preventiva e Social da Faculdade de Ciências Médicas da Universidade Estadual de Campinas (FCM/UNICAMP).

A amostra do inquérito foi obtida por intermédio de amostragem probabilística, estratificada, por conglomerados e em dois estágios: setor censitário e domicílio. No primeiro estágio, 50 setores censitários foram sorteados com probabilidade proporcional ao tamanho (número de domicílios). O sorteio foi sistemático, ordenando-se os setores pelo percentual de chefes que possuíam nível universitário, produzindo uma estratificação implícita por escolaridade do chefe de família.

O número de pessoas para compor a amostra foi obtido tendo em conta a situação correspondente à máxima variabilidade para a frequência dos eventos estudados ( $\mathrm{p}=0,50$ ), nível de $95 \%$ de confiança na determinação dos intervalos de confiança $(z=1,96)$, erro de amostragem entre 4 e 5 pontos percentuais e efeito de delineamento igual a 2, totalizando mil indivíduos em cada domínio de idade, a saber: adolescentes (10-19 anos), adultos (20-59 anos) e idosos (60 anos e mais). Esperando-se uma taxa de $80 \%$ de resposta, o tamanho da amostra foi corrigido para 1.250. Para alcançar esse tamanho de amostra em cada domínio, após atualização em campo dos mapas dos setores sorteados e elaboração da listagem de endereços, foram selecionados, de forma independente, 2.150, 700 e 3.900 domicílios para adolescentes, adultos e idosos, respectivamente. Nesta pesquisa, em cada domicílio, foram entrevistados todos os moradores da faixa etária selecionada para aquele domicílio 28.

As informações foram obtidas por questionário estruturado previamente testado em estudo piloto e aplicado em entrevistas domiciliares, realizadas por entrevistadores treinados e supervisionados. A integridade das entrevistas foi verificada em amostra aleatória de $5 \%$ dos participantes por meio de reentrevista domiciliar ou 
telefônica, e não mostrou discrepância com os dados originais.

Neste estudo foram utilizados os dados do inquérito referentes às pessoas com 60 anos ou mais, e a variável dependente foi o uso de ao menos um medicamento segundo prescrição. As informações sobre o uso de medicamentos foram obtidas mediante as seguintes perguntas: (1) "Você utilizou algum medicamento nos últimos três dias?” (2) “Quantos medicamentos? Quais?”. Para cada medicamento utilizado foi perguntado: (3) "Quem indicou esse medicamento para você?".

Na identificação dos medicamentos, quando possível, foi solicitado que o idoso apresentasse a embalagem do medicamento e/ou a prescrição médica para minimizar eventuais erros na anotação dos dados pelo entrevistador. Os medicamentos foram classificados de acordo com a Anatomical Therapeutic Chemical Code (ATC-2009) 29. Nessa classificação, eles são divididos de acordo com o grupo anatômico ou sistema em que atuam e suas propriedades químicas, terapêuticas e farmacológicas. Para identificar a composição dos medicamentos foi utilizado o Dicionário de Especialidades Farmacêuticas (DEF 2008/2009) 30. Para os medicamentos cujos nomes o entrevistado não soube referir, foi atribuído um código de não identificado; para os produtos que não constavam na ATC, foram criados códigos para identificá-los e para aqueles que não apresentavam um código específico na ATC, utilizou-se o código até o limite que possibilitou identificar o grupo, a classe ou a ação terapêutica.

Considerou-se automedicação o consumo, referido pelo idoso, de medicamentos sem prescrição por profissional habilitado (médico ou dentista), indicados por farmacêutico/balconista de farmácia, leigos (parente, amigo ou vizinho), por conta própria ou reaproveitados de terapias anteriores. Com base nas respostas obtidas, os participantes foram alocados em dois grupos, a saber: aqueles que relataram o consumo somente de medicamentos prescritos por médico ou dentista e aqueles que consumiram, simultaneamente, medicamentos prescritos e sem prescrição.

Para a análise dos fatores associados à automedicação, os seguintes conjuntos de variáveis independentes foram selecionados:

- Demográficas e socioeconômicas: sexo, idade, situação conjugal, cor da pele, escolaridade, número de residentes no domicílio, renda familiar per capita (em salários mínimos) e atividade ocupacional;

- Indicadores de condições de saúde e características relacionadas ao uso de serviços de saúde: percepção da própria saúde, morbidade referida nos 15 dias anteriores à pesquisa, relato de hiper- tensão, doença cardiovascular, diabetes, reumatismo/artrite/artrose, problemas de circulação (varizes, AVC), presença de transtorno mental comum (TMC), avaliada por meio do Self-Report Questionnarie (SRQ-20), procura por serviço ou profissional de saúde para problemas relacionados à saúde nos últimos 15 dias, consulta ao dentista nos últimos 12 meses, internação hospitalar no último ano e filiação a plano médico de saúde.

A análise de dados foi desenvolvida em duas etapas: inicialmente foi realizada análise bivariada pelo teste qui-quadrado com nível de 5\% de significância para verificar a associação entre as diversas variáveis e o uso de medicamentos sem prescrição. Também foram calculadas razões de prevalência (RP) ajustadas por sexo e idade e intervalos de 95\% de confiança (IC95\%). Nessa análise, as diferenças de gênero no consumo de medicamentos foram controladas, e a idade foi considerada variável preditora da terapia medicamentosa, já que a chance de usar algum tipo de fármaco aumenta desde a quarta década de vida13. Em seguida, foirealizadaanálisemúltiplapela regressão de Poisson com variância robusta 31 . No modelo múltiplo, o critério para inclusão foi a associação com o consumo de ao menos um medicamento sem prescrição, em nível inferior a 0,20 na análise bivariada e permaneceram no modelo apenas aquelas variáveis $\operatorname{com} \mathrm{p}<0,05$. As entrevistas foram digitadas em banco de dados desenvolvido com o uso do Epidata 3.1 (Epidata Assoc., Odense, Dinamarca) e as análises dos dados foram realizadas pela utilização do programa Stata 11.0 (Stata Corp., College Station, Estados Unidos), cujos procedimentos para análise de inquéritos populacionais incorporam os aspectos da amostragem complexa.

O estudo foi aprovado pelo Comitê de Ética da FCM/UNICAMP em adendo ao parecer $n^{\circ}$. 079/2007.

\section{Resultados}

Dos 1.515 idosos com respostas válidas para o uso de medicamento nos três dias que antecederam a pesquisa, 94,2\% responderam pessoalmente à entrevista. As demais entrevistas foram respondidas por cuidadores, parentes ou responsáveis, diante da impossibilidade do idoso responder, exceto questões subjetivas, como a percepção da saúde. Em relação à população estudada, 57,2\% eram mulheres, e a idade média foi de 69,9 anos (IC95\%: 69,3-70,6).

A prevalência do uso de medicamentos na população idosa nos três dias anteriores à pesquisa foi de 80,4\% (IC95\%: 77,6-83,2) e, entre os 
que referiram o uso (1.222), o consumo médio por idoso foi de 3,1 (IC95\%: 2,9-3,2) e, dentre esses, $24,8 \%$ referiram uso de ao menos cinco medicamentos. Entre os idosos que consumiram medicamentos, $1.116(91,1 \%)$ relataram ter consumido fármacos exclusivamente prescritos por médicos ou dentistas e 106 (8,9\%; IC95\%: 6,711,5) referiram consumo simultâneo de medicamentos prescritos e não prescritos. Não houve registro de idoso que tenha consumido exclusivamente medicamentos não prescritos nos três dias que antecederam a pesquisa.

Em relação aos medicamentos consumidos sem prescrição pelos idosos, os mais utilizados foram os medicamentos que atuam sobre o sistema nervoso central (dipirona - 25,7\%, Ginkgo biloba-9,6\%, paracetamol-8,8\% e AAS - 15,9\%), sistema musculoesquelético (diclofenaco-13\%), homeopáticos (6\%), aparelho digestivo e metabolismo (vitaminas e sais minerais $-4,1 \%$ e hioscina $-3,7 \%$ ) e fitoterápicos (3,4\%). O Ginkgo biloba foi avaliado separadamente dos demais fitoterápicos por apresentar um consumo diferenciado em estudo anterior realizado em Campinas 32.

A Tabela 1 apresenta a prevalência da automedicação, segundo variáveis demográficas e socioeconômicas dos idosos. Faixa etária, escolaridade e renda mensal per capita apresentaram-se associadas ao consumo de medicamentos não prescritos na análise simples $(\mathrm{p}<0,05)$. Após ajuste por sexo, observou-se redução significativa da automedicação com o aumento da idade. Também foi possível verificar aumento significativo do consumo sem prescrição com a ampliação da renda (RP ajustada por sexo e idade).

Em relação aos indicadores de condição de saúde e uso de serviços de saúde, hipertensão arterial, doença cardiovascular, diabetes, presença de doença crônica e procura por serviço nos 15 dias que antecederam a pesquisa, estiveram associadas à automedicação nos idosos na análise simples $(\mathrm{p}<0,05)$. Após ajuste por idade e sexo, as prevalências de automedicação apresentaram-se significativamente menores entre os hipertensos, diabéticos, naqueles que referiram presença de doença crônica e nos que procuraram serviço ou profissional de saúde nos 15 dias prévios. Entre portadores de doença cardiovascular, a menor prevalência esteve no limite da significância estatística $(\mathrm{RP}=0,64$; IC95\%: 0,40-1,00) (Tabela 2).

Os resultados da análise múltipla da regressão de Poisson são apresentados na Tabela 3. Foi possível verificar associação independente negativa entre a automedicação e a idade (80 anos e mais), hipertensão arterial referida, presença de uma ou mais doenças crônicas, uso de serviços de saúde nos 15 dias que antecederam a pesqui- sa, realização de consultas odontológicas no último ano e filiação a plano médico de saúde. Já a renda familiar per capita esteve positivamente associada à automedicação entre os idosos, ou seja, quanto maior a renda, maior o consumo de medicamentos não prescritos.

\section{Discussão}

A prevalência do uso de ao menos um medicamento não prescrito nos três dias que antecederam a pesquisa foi de $8,9 \%$ entre os idosos residentes em Campinas. Na investigação do uso de medicamentos, um dos mais importantes aspectos metodológicos refere-se ao período recordatório. Sabe-se que a prevalência do uso de medicamentos depende do período considerado ${ }^{33}$. Van der Geest \& Hardon ${ }^{34}$ destacam que medicamentos autoprescritos são particularmente mais sensíveis à dificuldade de recordar. Flores \& Mengue 35 também apontam o viés de memória em relação a medicamentos de uso esporádico, em geral não prescritos. Para Bertoldi et al. 33 , não há um período recordatório ideal para se investigar o uso medicamentos, uma vez que a seleção desse período depende do que está sendo recordado e da capacidade de a população respondente relembrar. Ainda, os autores destacam a tendência observada na literatura de que estudos sobre enfermidades agudas utilizam períodos recordatórios mais curtos, em comparação àqueles sobre enfermidades crônicas. Van den Brandt et al. 36 verificaram que a acurácia do recordatório do uso de medicamentos diminui com o aumento da idade e do número de medicamentos prescritos para uso crônico, e varia com o tipo de droga utilizada. Nesse sentido, o período recordatório utilizado neste estudo, considerando-se a população investigada e a autoprescrição dos medicamentos, parece adequado para descrever o evento estudado.

Diante do exposto, as prevalências encontradas foram comparadas apenas com trabalhos que consideraram um período recordatório igual ou inferior a uma semana. Flores \& Benvegnú 6 encontraram prevalência de $4 \%$ em Santa Rosa, no Rio Grande do Sul, variando de 3,8\% nas mulheres a 4,3\% em homens, inferiores àquelas observadas neste estudo, sem, contudo, verificar diferença estatisticamente significativa segundo gênero, corroborando os achados deste trabalho. Já estudo realizado em área urbana no Nordeste do Brasil verificou que $37,4 \%$ dos idosos usavam pelo menos um medicamento não prescrito 5 . Ambos os estudos sobre o perfil de utilização de medicamentos entre idosos também avaliaram o uso segundo prescrição e consideraram período 
Prevalência do consumo de ao menos um medicamento sem prescrição, segundo variáveis demográficas e socioeconômicas em pessoas com 60 anos ou mais. Inquérito de Saúde no Município de Campinas (ISA-Camp), 2008-2009.

\begin{tabular}{|c|c|c|c|c|}
\hline Variáveis e categorias & $\mathbf{n}$ & $\%$ (IC95\%) & Valor de $p$ * & $\mathrm{RP}_{\text {ajustada }}(\mathrm{IC} 95 \%)$ \\
\hline Sexo & & & 0,1735 & \\
\hline Masculino & 443 & $10,9(6,8-17,0)$ & & 1,00 \\
\hline Feminino & 779 & $7,6(5,7-9,9)$ & & $0,72(0,43-1,20)$ \\
\hline Faixa etária (anos) & & & $0,0025 * \star$ & \\
\hline $60-69$ & 622 & $11,4(8,2-15,7)$ & & 1,00 \\
\hline $70-79$ & 428 & $6,8(4,8-9,6)$ & & $0,60(0,40-0,89)$ \\
\hline 80 e mais & 172 & $4,8(2,7-8,4)$ & & $0,43(0,23-0,79)$ \\
\hline Situação conjugal & & & 0,8175 & \\
\hline Casado/União estável & 633 & $8,7(6,0-12,6)$ & & 1,00 \\
\hline Solteiro & 171 & $7,8(4,5-13,2)$ & & $1,02(0,58-1,79)$ \\
\hline Viúvo & 388 & $9,6(6,6-13,6)$ & & $1,86(1,14-3,06)$ \\
\hline Cor & & & 0,4471 & \\
\hline Branca & 945 & $8,5(6,7-10,6)$ & & 1,00 \\
\hline Não branca & 276 & $10,2(5,7-17,5)$ & & $1,11(0,70-1,76)$ \\
\hline Escolaridade (anos) & & & 0,0195 & \\
\hline $0-4$ & 812 & $7,2(5,5-9,4)$ & & 1,00 \\
\hline $5-8$ & 123 & $12,4(7,5-19,9)$ & & $1,55(0,98-2,45)$ \\
\hline 9 e mais & 286 & $11,7(7,6-17,7)$ & & $1,44(0,95-2,18)$ \\
\hline Número de residentes no domicílio & & & 0,4471 & \\
\hline $1-2$ & 674 & $9,8(7,5-12,7)$ & & 1,00 \\
\hline $3-4$ & 377 & $7,1(3,8-12,8)$ & & $0,69(0,40-1,20)$ \\
\hline 5 ou mais & 171 & $8,9(5,3-14,6)$ & & $0,90(0,50-1,63)$ \\
\hline Renda mensal per capita (SM) *** & & & 0,0034 ** & \\
\hline$\leq 1$ & 508 & $5,1(3,4-7,8)$ & & 1,00 \\
\hline$>1 e \leq 3$ & 484 & $10,4(7,5-14,1)$ & & $2,00(1,18-3,38)$ \\
\hline$>3$ & 230 & $13,4(8,2-21,0)$ & & $2,38(1,37-4,14)$ \\
\hline Atividade ocupacional & & & 0,0829 & \\
\hline $\operatorname{Sim}$ & 221 & $11,9(7,6-18,1)$ & & 1,00 \\
\hline Não & 1.000 & $8,2(6,1-10,8)$ & & $0,93(0,59-1,48)$ \\
\hline
\end{tabular}

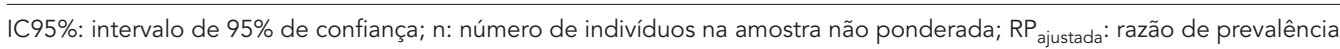
ajustada por sexo e idade; SM: salários mínimos.

* Valor de $\mathrm{p}$ do teste qui-quadrado;

** Teste de tendência linear;

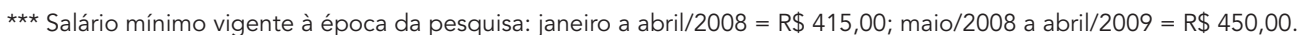

recordatório de sete dias. Marin et al. 17 verificou, em estudo realizado entre idosos residentes em área de abrangência de uma unidade de saúde da família em cidade do interior paulista, que 36,9\% faziam uso de algum tipo de fármaco sem indicação médica no dia da entrevista. Pelicioni \& César 12, utilizando um período recordatório de três dias verificaram, com base em dados de inquérito domiciliar de saúde realizado em quatro áreas do Estado de São Paulo (ISA-SP), prevalências de $13 \%$ e $16 \%$ para a população masculina e feminina de 60-69 anos, respectivamente, e de $9 \%$ nos homens e $10 \%$ nas mulheres na faixa etária de 70 anos ou mais, percentual semelhante ao verificado neste estudo que contou com uma população cuja idade média esteve próxima aos 70 anos de idade. Quando comparados os gêneros, o consumo de medicamentos sem indicação médica ou odontológica não apresentou diferença estatística em relação ao sexos. No Município de Campinas, Perrone et al. 27 com dados de inquérito realizado em 2001-2002, encontraram prevalência de automedicação de 5,2\% entre os homens e $10,2 \%$ em mulheres de 60 anos ou mais, com 
Prevalência do consumo de ao menos um medicamento sem prescrição, segundo indicadores de condição de saúde e uso de serviços de saúde em pessoas com 60 anos ou mais. Inquérito de Saúde no Município de Campinas (ISA-Camp), 2008-2009.

\begin{tabular}{|c|c|c|c|c|}
\hline Variáveis e categorias & $\mathrm{n}$ & \% (IC95\%) & Valor de $p$ * & $\mathrm{RP}_{\text {ajustada }}(\mathrm{IC95 \% )}$ \\
\hline Percepção da própria saúde & & & 0,419 & \\
\hline Excelente/Muito boa/Boa & 999 & $9,5(7,3-12,1)$ & & 1,00 \\
\hline Ruim/Muito ruim & 149 & $6,5(2,3-17,3)$ & & $0,68(0,26-1,75)$ \\
\hline Morbidade nos últimos 15 dias & & & 0,816 & \\
\hline Não & 867 & $9,0(7,0-11,4)$ & & 1,00 \\
\hline Sim & 354 & $8,5(5,0-14,1)$ & & $0,96(0,60-1,53)$ \\
\hline \multicolumn{5}{|l|}{ Relato das seguintes doenças/ } \\
\hline \multicolumn{5}{|l|}{ Condições crônicas ** } \\
\hline Hipertensão & 800 & $5,9(4,1-11,5)$ & $<0,001$ & $0,44(0,30-0,64)$ \\
\hline Doença cardiovascular & 260 & $5,6(3,5-8,9)$ & 0,016 & $0,64(0,40-1,00)$ \\
\hline Diabetes & 322 & $4,9(2,8-8,6)$ & 0,003 & $0,49(0,30-0,80)$ \\
\hline Artrite/Reumatismo/Artrose & 369 & $10,0(6,9-14,3)$ & 0,299 & $1,36(0,96-1,92)$ \\
\hline Problemas de circulação & 370 & $6,6(4,4-9,9)$ & 0,061 & $0,76(0,51-1,13)$ \\
\hline Número de doenças crônicas & & & $<0,001$ & \\
\hline Nenhuma & 97 & $27,2(16,6-41,2)$ & & 1,00 \\
\hline Uma ou mais & 1.105 & $7,2(5,6-9,2)$ & & $0,30(0,20-0,44)$ \\
\hline SQR-20 & & & 0,229 & \\
\hline Negativo & 994 & $9,4(7,3-12,1)$ & & 1,00 \\
\hline Positivo & 226 & $6,4(3,1-12,7)$ & & $0,78(0,39-1,59)$ \\
\hline Procura de serviço ou profissional de & & & 0,004 & \\
\hline \multicolumn{5}{|l|}{ saúde para atendimento nos últimos } \\
\hline \multicolumn{5}{|l|}{15 dias } \\
\hline Não & 873 & $10,2(8,0-12,9)$ & & 1,00 \\
\hline Sim & 349 & $5,4(3,1-9,3)$ & & $0,54(0,34-0,85)$ \\
\hline Internação hospitalar no último ano & & & 0,114 & \\
\hline Não & 1.027 & $9,4(7,1-12,3)$ & & 1,00 \\
\hline Sim & 195 & $5,8(3,2-10,6)$ & & $0,66(0,34-1,21)$ \\
\hline Consulta odontológica nos últimos & & & 0,197 & \\
\hline \multicolumn{5}{|l|}{12 meses } \\
\hline Não & 797 & $9,6(6,9-13,2)$ & & 1,00 \\
\hline Sim & 425 & $7,5(5,4-10,3)$ & & $0,68(0,46-1,01)$ \\
\hline Plano odontológico & & & 0,267 & \\
\hline Não & 1.143 & $8,6(6,5-11,3)$ & & 1,00 \\
\hline Sim & 79 & $11,7(6,6-20,0)$ & & $1,22(0,72-2,07)$ \\
\hline Plano médico de saúde & & & 0,140 & \\
\hline Não & 611 & $10,0(7,1-13,9)$ & & 1,00 \\
\hline Sim & 610 & $7,7(5,7-10,3)$ & & $0,83(0,59-1,19)$ \\
\hline
\end{tabular}

associação estatística significativa entre os sexos. Comparando as proporções de autoprescrição verificadas para as diferentes grupos de idade, os autores destacam provável racionalidade na prática de automedicação nas faixas etárias em que as pessoas são mais vulneráveis e os problemas de saúde mais frequentes (crianças e idosos).
Situação conjugal, cor da pele, número de residentes no domicílio e realização de atividade ocupacional referidas pelo entrevistado, não estiveram associadas à automedicação. Nos estudos com período recordatório semelhante consultados, tais variáveis não foram avaliadas. 
Modelo de regressão multivariada de Poisson para automedicação em pessoas com 60 anos ou mais. Inquérito de Saúde no Município de Campinas (ISA-Camp), 2008-2009.

\begin{tabular}{|c|c|c|c|}
\hline Variáveis & $\mathrm{RP}_{\text {ajustada }}(\mathrm{IC} 95 \%)$ & Valor de $p$ & Erro-padrão \\
\hline \multicolumn{4}{|l|}{ Faixa etária (anos) } \\
\hline $70-79$ & $0,70(0,47-1,05)$ & 0,083 & 0,14 \\
\hline 80 e mais & $0,38(0,21-0,70)$ & 0,003 & 0,12 \\
\hline \multicolumn{4}{|l|}{ Renda familiar per capita (SM) * } \\
\hline$>1$ e $\leq 3$ & $2,08(1,26-3,44)$ & 0,005 & 0,52 \\
\hline$>3$ & $3,04(1,73-5,33)$ & $<0,001$ & 0,85 \\
\hline Hipertensão arterial & $0,59(0,36-0,96)$ & 0,033 & 0,14 \\
\hline Presença de doença crônica & $0,47(0,29-0,75)$ & 0,002 & 0,11 \\
\hline Uso de serviços de saúde nos 15 dias que & $0,57(0,34-0,94)$ & 0,028 & 0,14 \\
\hline \multicolumn{4}{|l|}{ antecederam a pesquisa } \\
\hline Consulta odontológica no último ano & $0,60(0,40-0,88)$ & 0,011 & 0,12 \\
\hline Filiação a plano médico de saúde & $0,63(0,43-0,91)$ & 0,014 & 0,11 \\
\hline
\end{tabular}

IC95\%: Intervalo de 95\% de confiança; $\mathrm{RP}_{\text {ajustada }}$ razão de prevalência ajustada por meio de regressão múltipla de Poisson (1.201 indivíduos foram incluídos no modelo final); SM: salários mínimos.

* Salário mínimo vigente à época da pesquisa: janeiro a abril/2008 = R\$ 415,00; maio/2008 a abril/2009=R\$450,00.

Em relação ao consumo de medicamentos sem prescrição, segundo indicadores de condição de saúde e uso de serviços de saúde, na análise ajustada por idade e sexo, hipertensão, diabetes e a procura de serviço ou profissional de saúde para atendimento nos últimos 15 dias estiveram inversamente associadas à automedicação. $\mathrm{O}$ Município de Campinas possui uma rede de atenção básica organizada para atender as necessidades da população nesse nível de atenção. Cerca de 52\% (IC95\%: 44,2-60,2) dos idosos do município não possuem plano privado de saúde sendo, portanto, exclusivamente dependentes do sistema público de saúde 37 . Os medicamentos para tratamento de doenças crônicas como hipertensão e diabetes são oferecidos gratuitamente na rede básica garantindo o acesso ao idoso portador de tais enfermidades. Ainda foi possível verificar associação independente negativa entre a automedicação e a idade de 80 anos e mais. A proporção de automedicação também foi significativamente menor entre os idosos que referiram filiação a plano médico de saúde. Em relação à renda, encontrou-se associação independente com um gradiente positivo, ou seja, quanto maior a renda, maior a prevalência de automedicação.

Estudo de Coelho Filho et al. 5 verificou maior chance de utilização de medicamentos exclusivamente prescritos entre idosos que referiram visita a serviços de saúde, presença de doença crônica, melhor nível socioecônomico e idade acima de 75 anos. Considerando-se o nível so- cioecônomico menos favorecido, os autores verificaram ainda que a proporção de idosos usando medicamentos prescritos foi menor, diferentemente do que foi verificado no presente estudo.

Neste estudo, não houve registro de idosos que fizeram uso apenas de medicamentos não prescritos e sim uso simultâneo de medicamentos prescritos e não prescritos. Assim, é possível que para os idosos residentes em Campinas, o uso de fármacos que dispensam a receita seja entendido como uma prática corriqueira, sem prejuízos à saúde. Todavia, o uso concomitante de tais medicamentos com outras drogas prescritas, associado a fatores inerentes a cada indivíduo e considerando-se seu estado de saúde particular, requer avaliação dos riscos à saúde, especialmente no que tange às intoxicações e interações medicamentosas. A automedicação é um elemento do autocuidado ${ }^{22}$, mas para que não traga complicações ao indivíduo e à saúde pública, é fundamental que seu manejo seja subsidiado por informações suficientes, que garantam a automedicação responsável, definida como " a prática dos cidadãos em tratar seus próprios sintomas e males menores com medicamentos aprovados pelas autoridades sanitárias, disponíveis sem a prescrição ou receita médica e seguros e eficazes quando usados segundo as instruções" 22 (p. 3). Nesse contexto, entre os idosos, a orientação do farmacêutico, enquanto profissional com competência para realizar aconselhamento sobre os medicamentos que fornece, é fundamental e 
pode contribuir significativamente para do uso racional de medicamentos.

Foi possível verificar que o acesso a serviços de saúde, bem como a presença de ao menos uma doença crônica reduzem a prática de automedicação. Particularmente em relação à hipertensão arterial, a associação independente permite sugerir algumas hipóteses: por se tratar de doença crônica com alta prevalência entre os idosos e diante da disponibilidade de atendimento e da oferta de medicamentos na rede pública, essa enfermidade leva o indivíduo ao serviço de saúde com maior frequência para controle dos níveis pressóricos, busca de medicação e exames periódicos. Nesse sentido, o idoso hipertenso tem mais acesso às informações de saúde pelo maior contato com profissionais de saúde. Além disso, pessoas portadoras de enfermidades crônicas em geral, como o diabetes, contam com acompanhamento médico e são usuárias mais assíduas dos serviços de saúde.

O número médio de medicamentos consumidos pelos idosos residentes em Campinas está de acordo com o que vem sendo apontado pela literatura $5,6,9,17,35$. O fato de cerca de $25 \%$ dos idosos entrevistados nos três dias anteriores à entrevista estarem usando cinco ou mais medicamentos diferentes, confirma que o uso simultâneo de diversas especialidades farmacêuticas é uma prática comum entre eles. Na Itália, Pizzuti et al. 38 verificaram que $45,3 \%$ dos idosos utilizavam quatro ou mais medicamentos diferentes por dia. No estudo de Flores \& Benvegnú 6 , mais de $30 \%$ dos entrevistados referiram uso diário de pelo menos três medicamentos. Marin et al. 17 verificaram em estudo realizado no Município de Marília (São Paulo) que 29,5\% dos idosos estavam fazendo uso de três ou quatro medicamentos na ocasião da entrevista e que $4 \%$ utilizavam oito ou mais.

Entre os medicamentos sem prescrição mais utilizados, destacaram-se os analgésicos antipiréticos, e a dipirona apresentou a maior proporção de consumo. Segundo Arrais 39, tal achado tem sido destacado desde a década de 80 no Brasil e em estudos internacionais como uma das classes farmacológicas de maior consumo na população. O diclofenaco foi o anti-inflamatório não prescrito mais utilizado pelos idosos; a proporção de uso de Ginkgo biloba sem indicação médica foi de 9,6\%. A ação combinada dos diferentes princípios ativos presentes no extrato promove o aumento do suprimento sanguíneo cerebral pela vasodilatação e redução da viscosidade do sangue, além de diminuir a densidade de radicais livres de oxigênio nos tecidos nervosos. No entanto, seus benefícios à saúde humana, em particular sobre a demência e deficiên- cia cognitiva, não foram totalmente estabelecidas 40 . Estudo recente verificou melhora de cognição entre pacientes com Alzheimer e demência vascular ou mista associada do uso do Ginkgo biloba ${ }^{41}$. Puppo \& Silva 42 alertam para os riscos das possíveis interações entre o Ginkgo biloba e o AAS, comumente utilizado pelos idosos, porque essa associação, por aumentar a inibição da agregação plaquetária, pode causar hemorragias, representando riscos para a saúde do idoso. Cascaes et al. 43 destacam que os principais medicamentos sem a orientação de profissionais de saúde consumidos por esse grupo etário são plantas medicinais e medicamentos de venda livre no manejo dos problemas de saúde que consideram simples. Neste estudo, na avaliação da distribuição das especialidades farmacêuticas utilizadas sem prescrição pelos idosos, frente ao motivo de uso de tais medicamentos (cefaleia, resfriado comum ou gripe, dor muscular e deficiências vitamínicas entre outros), observou-se que na maioria dos casos tratava-se de fármacos de valor intrínseco não elevado.

De acordo com Coelho Filho et al. 5, diferentes definições para medicamentos não prescritos dificultam a comparação com estudos realizados em países desenvolvidos. Na língua inglesa a terminologia non-prescription medication ou over-the-counter medication se refere ao uso de medicamento que não requer receita médica. Já nos trabalhos nacionais é usual considerar medicamento não prescrito todo aquele não prescrito por médico ou profissional de saúde habilitado, independentemente da inclusão de fármacos para os quais o uso necessite ou não de receita médica. Essa questão deve ser levada em conta, pois traz implicações nos resultados dos estudos e reflexões importantes para as ações de saúde pública.

Diferenças no padrão de uso de medicamentos e na prevalência de automedicação podem ser parcialmente atribuídas a especificidades das populações, ao estado de saúde, à utilização de serviços e modelo de atenção à saúde, bem como a características demográficas, sociais e culturais ligadas ao consumo de medicamentos. Além disso, o período recordatório, as definições utilizadas quanto ao evento estudado (consumo exclusivo de qualquer medicamento não prescrito ou uso simultâneo de prescritos e não prescritos; automedicação esperada ou não esperada) entre outros aspectos, devem ser considerados, sugerindo cautela nas comparações. O menor consumo de medicamentos sem prescrição entre idosos no Município de Campinas pode ser atribuído a alguns desses fatores.

Entre as limitações deste estudo, destaca-se o fato de que o período recordatório de três dias 
pode reduzir a prevalência de automedicação, visto que quanto mais passa o tempo (15 ou 30 dias), maior a probabilidade de uso eventual de algum fármaco. Os medicamentos de uso contínuo são igualmente contados em qualquer período de tempo. Porém, deve-se considerar que um espaço de tempo maior também poderia gerar um viés de memória em relação aos medicamentos de uso esporádico utilizados, promovendo subdeclaração, especialmente de fármacos usados para enfermidades de menor gravidade 32,33. Deve-se considerar, ainda, que este estudo utilizou dados de um amplo inquérito de saúde, o que pode levar a resultados diferentes daqueles obtidos num inquérito específico sobre o tema, entretanto, verifica-se coerência dos achados do presente estudo com resultados anteriores, tanto em relação ao perfil sociodemográfico, quanto às especialidades farmacêuticas sem prescrição mais consumidas pela população estudada.

O uso de medicamentos, induzido por campanhas publicitárias que os apresentam como bem de consumo e não como um insumo essencial às ações de promoção, prevenção e recuperação da saúde, sem o esclarecimento sobre os riscos associados ao seu uso, e atendendo à lógica de mercado, também favorece a prática da automedicação. A automedicação é uma prática generalizada, aceita em muitos países, que pode não provocar danos à saúde se realizada de forma responsável e assistida, no entanto, pode mascarar doenças evolutivas e provocar reações adversas 21 . Vale ressaltar que a prevalência de reação adversa à droga é três vezes maior em idosos do que em jovens, apresentando incidência anual de 26 por mil leitos de pacientes hospitalizados 44 .

Sobretudo entre os idosos, a automedicação precisa de ser auxiliada por profissionais de saúde, permitindo a identificação de problemas que necessitem de avaliação de profissional qualificado e a conscientização acerca de uma farmacoterapia racional, já que os riscos de tal prática estão associados ao grau de informação sobre os medicamentos.

\section{Conclusão}

Os resultados do estudo mostram baixa prevalência de automedicação entre os idosos e apontam que as apresentações não prescritas mais utilizadas neste segmento são medicamentos de venda livre, sugerindo uma possível observância de critérios técnicos no cuidado, bem como a qualidade da assistência farmacêutica oferecida aos idosos pelo município. O estudo revelou ainda que, em Campinas, a utilização de serviços de saúde, a realização de consultas odontológicas e a filiação a plano médico de saúde restringiram a prática da automedicação entre os idosos. Diante da importância que se reveste o tema e da necessidade de sua melhor compreensão, estudos como este podem ser utilizados como ferramenta em diferentes localidades, para subsidiar a promoção do uso racional de medicamentos neste segmento populacional.

\section{Resumo}

O objetivo foi avaliar a prevalência e fatores associados à automedicação em idosos e identificar os principais fármacos consumidos sem prescrição. Estudo transversal de base populacional, com amostra estratificada por conglomerados e em dois estágios realizado em Campinas, São Paulo, Brasil, em 2008-2009. Dos 1.515 idosos, 80,4\% referiram uso de ao menos um medicamento nos três dias anteriores à pesquisa. Desses, 91,1\% relataram consumo exclusivo de medicamentos prescritos e o restante (8,9\%), uso simultâneo de prescritos e não prescritos. Após ajuste, idade $\geq 80$ anos, hipertensão arterial, presença de doenças crônicas, uso de serviços de saúde, realização de con- sultas odontológicas e filiação a plano médico de saúde estiveram associadas negativamente, e renda per capita, positivamente à automedicação. Os fármacos sem prescrição mais consumidos foram dipirona, AAS, diclofenaco, Ginkgo biloba, paracetamol e homeopáticos. Sobretudo entre idosos, a assistência farmacêutica deve ser priorizada para evitar o uso incorreto de medicamentos e garantir o acesso aos fármacos necessários ao tratamento.

Automedicação; Uso de Medicamentos; Farmacoepidemiologia; Saúde do Idoso 


\section{Colaboradores}

M. A. Oliveira e P. M. S. B. Francisco realizaram a proposta do artigo, revisão de literatura, análise dos dados e redação do texto. K. S. Costa e M. B. A. Barros colaboraram na discussão e na revisão do texto.

\section{Agradecimentos}

Ao Conselho Nacional de Desenvolvimento Científico e Tecnológico (CNPq; processo n ${ }^{\circ}$. 409747/2006-8) pelo financiamento da pesquisa e pela bolsa de produtividade de M. B. A. Barros. À Fundação de Amparo à Pesquisa do Estado de São Paulo (FAPESP), pela bolsa de pósdoutorado recebida por P. M. S. B. Francisco.

\section{Referências}

1. Lloyd-Sherlock P. Population ageing in developed and developing regions: implications for health policy. Soc Sci Med 2000; 51:887-95.

2. Lebrão ML, Laurenti R. Saúde, bem-estar e envelhecimento: o estudo SABE no município de São Paulo. Rev Bras Epidemiol 2005; 8:127-41.

3. Veras R. Envelhecimento populacional contemporâneo: demandas, desafios e inovações. Rev Saúde Pública 2009; 43:548-54.

4. Ramos LR, Garcia JT. Terapêutica medicamentosa no idoso. In: Prado FC, Ramos J, Valle JR, organizadores. Atualização terapêutica. 21ạ Ed. São Paulo: Editora Artes Médicas; 2003. p.555-8.

5. Coelho Filho JM, Marcopito LF, Castelo A. Perfil de utilização de medicamentos por idosos em área urbana do Nordeste do Brasil. Rev Saúde Pública 2004; 38:557-64.

6. Flores VB, Benvegnú LA. Perfil de utilização de medicamentos em idosos da zona urbana de Santa Rosa, Rio Grande do Sul, Brasil. Cad Saúde Pública 2008; 24:1439-46.

7. Ribeiro AQ, Rozenfeld S, Klein CH, César CC, Acúrcio FA. Inquérito sobre uso de medicamentos por idosos aposentados, Belo Horizonte, MG. Rev Saúde Pública 2008; 42:724-32.
8. Rozenfeld S. Prevalência, fatores associados e mau uso de medicamentos entre idosos: uma revisão. Cad Saúde Pública 2003; 19:717-24.

9. Mosegui GBG, Rozenfeld S, Veras RPV, Vianna CMM. Avaliação da qualidade do uso de medicamentos em idosos. Rev Saúde Pública 1999; 33:437-44.

10. Linjakumpu T, Hartikainen S, Klaukka T, Veijola J, Kivelä SL, Isoaho R. Use of medications and polypharmacy are increasing among the elderly. J Clin Epidemiol 2002; 55:809-17.

11. Loyola Filho AI, Uchoa E, Lima-Costa MF. Estudo epidemiológico de base populacional sobre uso de medicamentos entre idosos na Região Metropolitana de Belo Horizonte, Minas Gerais, Brasil. Cad Saúde Pública 2006; 22:2657-67.

12. Pelicioni AF, César CLG. Consumo de medicamentos. In: Cesar CLG, Carandina L, Alves MCGP, Barros MBA, Goldbaum M, organizadores. Saúde e condição de vida em São Paulo: Inquérito Multicêntrico de Saúde no Estado de São Paulo - ISA-SP. São Paulo: Faculdade de Saúde Pública, Faculdade de São Paulo; 2005. p. 199-212. 
13. Rocha CH, Oliveira APS, Ferreira C, Faggiani FT, Schroeter G, Souza ACA, et al. Adesão à prescrição médica em idosos de Porto Alegre, RS. Ciênc Saúde Coletiva 2008; 13 Suppl:703-10.

14. Loyola Filho AI, Uchoa E, Guerra HL, Firmo JOA, Lima-Costa MF. Prevalência e fatores associados à automedicação: resultados do projeto Bambuí. Rev Saúde Pública 2002; 36:55-62.

15. Loyola Filho AI, Uchoa E, Firmo JOA, Lima-Costa MF. Estudo de base populacional sobre o consumo de medicamentos entre idosos: Projeto Bambuí. Cad Saúde Pública 2005; 21:545-53.

16. Flores LM, Mengue SS. Uso de medicamentos por idosos em região do sul do Brasil. Rev Saúde Pública 2005 ; 39:924-9.

17. Marin MJS, Cecílio LCO, Perez AEWUF, Santella F, Silva CBA, Gonçalves Filho JR, et al. Caracterização do uso de medicamentos entre idosos de uma unidade do Programa Saúde da Família. Cad Saúde Pública 2008; 24:1545-55.

18. Brasil. Portaria ${ }^{\circ}$. 3916/MS/GM, de 30 de outubro de 1998. Aprovar a Política Nacional de Medicamentos. Diário Oficial da União 1998; 10 nov.

19. Sá MB, Barros JAC, Sá MPBO. Automedicação em idosos na cidade de Salgueiro-PE. Rev Bras Epidemiol 2007; 10:75-85.

20. Arrais PSD, Coelho HLL, Batista MCDS, Carvalho ML, Righi RE, Arnau JM. Perfil da automedicação no Brasil. Rev Saúde Pública 1997; 31:71-7.

21. Bortolon PC, Medeiros EFF, Naves JOS, Karnikowski MGO, Nóbrega OT. Análise do perfil de automedicação em mulheres idosas brasileiras. Ciênc Saúde Coletiva 2008; 13:1219-26.

22. World Health Organization. The role of the pharmacist in self-care and self-medication. Report of the 4th WHO Consultive Group on the role of the pharmacist. Geneva: World Health Organization; 1998.

23. Vilarino JF, Soares IC, Silveira CM, Rödel APP, Bortoli R, Lemos RR.. Perfil da automedicação em município do Sul do Brasil. Rev Saúde Pública 1998; 32:43-9.

24. Naves JOS, Castro LLC, Carvalho CMS, MerchanHamann E. Automedicação: uma abordagem qualitativa de suas motivações. Ciênc Saúde Coletiva 2010; 15 Suppl 1:1751-62.

25. Goh LY, Vitry AI, Semple SJ, Esterman A, Luszcz M. Self-medication with over-the-counter drugs and complementary medications in South Australia's elderly population. BMC Complement Altern Med 2009; 9:42.

26. Qato DM, Alexander GC, Conti RM, Johnson M, Schumm P, Lindau ST. Use of prescription and over-the-counter medications and dietary supplements among older adults in the United States. JAMA 2008; 300:2867-78.

27. Perrone AMF, Molina MC, Bertonha MEAM, Nativio J, Barros MBA. Uso de medicamentos. In: Barros MBA, César CLG, Carandina L, Goldbaum M, organizadores. As dimensões da saúde: inquérito populacional em Campinas, SP. São Paulo: Aderaldo \& Rothschild; 2008. p. 218-29.

28. Alves MCGP. ISA-Campinas 2008/09: plano de amostragem. http://www.fcm.unicamp.br/cen tros/ccas/arquivos/plano_de_amostragem.pdf (acessado em 16/Abr/2011).
29. World Health Organization. ATC/DDD index 2009. http://www.whocc.no/atcddd/indexdatabase/ (acessado em 18/Mai/2009).

30. Dicionário de especialidades farmacêuticas 2008/09. 37a Ed. 2008/09. Editora: Epub; 2009.

31. Barros AJ, Hirakata VN. Alternatives for logistic regression in cross-sectional studies: an empirical comparison of models that directly estimate the prevalence ratio. BMC Med Res Methodol 2003; $3: 21$.

32. Costa KS, Barros MBA, Francisco PMSB, César CLG, Goldbaum M, Carandina L. Utilização de medicamentos e fatores associados: um estudo de base populacional no Município de Campinas, São Paulo, Brasil. Cad Saúde Pública 2011; 27:649-58.

33. Bertoldi AD, Barros AJD, Wagner A, Dennis RD, Hallal PC. A descriptive review of the methodologies used in household surveys on medicine utilization. BMC Med Res Methodol 2008; 8:222.

34. Van der Geest S, Hardon A. Drugs use: methodological suggestions for field research in developing countries. Health Policy Plan 1988; 3:152-8.

35. Flores LM, Mengue SS. Uso de medicamentos por idosos em região do sul do Brasil. Rev Saúde Pública 2005 ; 39:924-9.

36. Van den Brandt PA, Petri H, Dorant E, Goldbohm RA, Van de Crommert S. Comparision of questionnaire information and pharmacy data on drug use. Pharm Weekbl Sci 1991; 13:91-6.

37. Barros MBA, Francisco PMSB, Lima MG, César CLG. Social inequalities in health among elderly. Cad Saúde Pública 2011; 27 Suppl 2:S198-208.

38. Pizzuti R, Caffari B, Binkin N, Gruppo ARGENTO. La prescrizione di farmaci nell'anziano: risultati dello Studio Argento. Ig Sanita Pubbl 2006; 62: 11-26.

39. Arrais PSP. Medicamentos: consumo e reações adversas: um estudo de base populacional. Fortaleza: Edições UFC; 2009.

40. Birks J, Grimley Evans J. Ginkgo biloba for cognitive impairment and dementia. Cochrane Database Syst Rev 2009; (1):CD003120.

41. Weinmann S, Roll S, Schwarzbach C, Vauth C, Willich SN. Effects of Ginkgo biloba in dementia: systematic review and meta-analysis. BMC Geriatr 2010; 10:14.

42. Puppo E, Silva CP. Levantamento do perfil medicamentoso e frequência de associações entre o Ginkgo (Ginkgo biloba L.) e ácido acetilsalicílico, em usuários atendidos pela FarmaUSCS de São Caetano do Sul. Rev Ciênc Farm Básica Apl 2008; 29:53-8.

43. Cascaes EA, Falchetti ML, Galato D. Perfil da automedicação em idosos participantes de grupos da terceira idade de uma cidade do sul do Brasil. ACM Arq Catarin Med 2008; 37:63-9.

44. Garcia JT. Uso de medicamentos. In: Ramos LR, Cendoroglo MS, organizadores. Geriatria e gerontologia. Barueri: Editora Manole; 2005. p. 27-36.

Recebido em 06/Jun/2011

Versão final reapresentada em 21/Set/2011

Aprovado em 03/Out/2011 